\title{
El contexto político en el momento de la creación del Instituto Andaluz del
} Patrimonio Histórico

\author{
Javier Torres Vela \\ Presidente del Parlamento Andaluz \\ Consejero de Cultura de la Junta de \\ Andalucía (1984-1990)
}

\begin{abstract}
Estar juntos hoy, con ocasión del décimo aniversario del Instituto Andaluz del Patrimonio Histórico, quien firmó su decreto de creación y quien es en la actualidad responsable de su andadura es síntoma de que la institución sigue funcionando muy bien. Pero eso es mérito, sobre todo, de todos aquellos que han estado trabajando aquí durante estos diez años.
\end{abstract}

Seguramente no tenga ninguna importancia para ustedes -y yo comprendo que no la tenga- pero es la primera vez, desde que salí de la Consejería de Cultura, que voy a reflexionar en público sobre una etapa de mi gestión. Nunca lo he querido hacer, porque pienso que en la vida política uno va sucediendo etapas y debe saber pasar las páginas sin mirar demasiado atrás, debe huir de la tentación de comparar -para no caer en la vanidad- lo que uno ha hecho con lo que han venido haciendo los demás. En este sentido, un maestro universitario me recomendó, justamente cuando salía de la Consejería de Cultura, una terapia: que durante algunos meses no leyera ni siquiera las páginas de cultura de los periódicos, e iniciará un nuevo horizonte. Les puedo decir que he sido fiel a esa consideración, y he dejado que la valoración de mi etapa la hicieran los ciudadanos que votan en las elecciones, los profesionales que pudieron colaborar... y ustedes hoy, diez años después.

Pero cuando Román Fernández-Baca me llama hace algún tiempo, y me invita a dar una charla en la apertura de una Jornada sobre el X Aniversario del Instituto Andaluz del Patrimonio Histórico, mi primera tentación, por lo que les acabo de decir, fue negarme. Pero después hubo una segunda reflexión, que me llevó a estar hoy aquí. De alguna manera, se lo debía. Se lo debía a Román y se lo debía a mucha gente, a algunos de los cuales veo por aquí, políticos unos, profesionales o amigos otros, gente común que en una etapa que yo calificaría de apasionante, de complicada, de difícil, me ayudaron a construir una política, una política en el sentido noble de la palabra, una política que trataba de administrar los bienes culturales, el Patrimonio histórico, materias en las que éramos novatos. Aquella política, pues, se empezaba a hacer casi desde cero, se inventó, o, mejor dicho, la inventaron ellos, porque yo únicamente fui en ese sentido un transmisor que dio el apoyo político para poder hacerlo.

Por lo tanto, como decía, estoy aquí porque se lo debía, a Román y a otros muchos que citaré al final. Aquel grupo humano -y en esto no hago un ejercicio de autosatisfacción personal- ayudó a construir una política, la política más avanzada y más innovadora que en el terreno de los bienes culturales se puso en marcha en España, y se hizo -les voy a decir también una afirmación previa- sin que estuviese pensada, sin un proyecto claro. Todo se desarrolló en un proceso largo de maduración, con enfrentamientos y tropiezos, y dejando alguna que otra herida en el proceso.

Pero antes de contarles ese proceso, antes de contarles algunas anécdotas (que el Instituto Andaluz del Patrimonio Histórico, por ejemplo, no estaba ni siquiera en el primer borrador del Plan General de Bienes Culturales, no estaba en el primer documento que yo elaboro en 1986 sobre las Instituciones básicas de la Comunidad) les haré una advertencia previa: no esperen de mí -he visto en el programa de las Jornadas que hay una gran cantidad de expertos nacionales e internacionales que vienen a reflexionar- una conferencia sobre el estado actual de la investigación o de la política de investigación y de conservación del Patrimonio Histórico, porque no soy experto en esa materia. Yo soy un matemático que se socializa en la política bajo la dictadura, alcanzando un compromiso político y moral que le lleva a militar en el Partido Socialista desde hace ya casi treinta años. Soy un político que en un período de su vida y de su responsabilidad política se ocupó de la Consejería de Cultura, y, por lo tanto, no puede ofrecer hoy una severa y documentada conferencia sobre las razones que impelieron a la Consejería de Cultura a crear un departamento de I+D en materia de Patrimonio Histórico que se denominó Instituto Andaluz del Patrimonio Histórico.

Permítanme que recurra entonces a mi memoria y que describa el proceso, vivido en Andalucía de 1984 a 1990, por el que se sientan las bases de lo que fue el Plan General de Bienes Culturales y con él la creación del Instituto Andaluz del Patrimonio Histórico. Y lo voy a hacer tal y como les he dicho, con un recurso a la memoria. He preparado una intervención sobre notas de lo que ha quedado en mi recuerdo de aquel periodo; sólo he consultado algunos documentos: el Decreto de creación del Instituto, el documento del Plan General de Bienes Culturales y la presentación que yo mismo hice del Plan en el Parlamento de Andalucía allá por 1989, en la que dimos a conocer el I 


\section{Recuerdo aquel periodo, los años del voluntarismo, como los años más duros, los más difíciles de toda mi etapa en la Consejería y probablemente en la política.}

Plan General de Bienes Culturales, aprobado por el Parlamento por unanimidad.

El Plan General de Bienes Culturales y el Instituto Andaluz del Patrimonio Histórico se presentan y crean respectivamente en la etapa y en el año final de mi periodo como Consejero de Cultura de la Junta de Andalucía, que acaba en los primeros meses de 1990. Es decir, tanto el Plan como la creación del Instituto son consecuencia, son resultado de una política, de un largo proceso de experiencia, de prueba y de error, de reflexión, de maduración, no son el avance de un proyecto -eso vendría después, con el seguimiento de otros y la culminación, en este momento, por Carmen Calvo. Así lo reconozco yo mismo, y perdónenme la autocita, en el Parlamento de Andalucía en junio de 1989 al presentar el Plan General de Bienes Culturales: "El Plan se inserta en la experiencia vivida y contrastada día a día en Andalucía desde 1984, año en que nuestra Comunidad asume las plenas competencias".

Justamente como recuerdo de esa experiencia vivida voy hablarles a continuación.

Comenzaré llevando al punto de partida el título propuesto para mi conferencia "El contexto político en el momento de la creación del Instituto", preguntándome cómo se inicia esa experiencia que concluye con la creación del Instituto Andaluz del Patrimonio Histórico.

Mi llegada a la Consejería de Cultura en 1984, en marzo de 1984, coincide con dos hechos que tienen una especial significación política para el tema de que vamos a hablar. El primero de ellos es la salida de Rafael Escudero de la Presidencia de la Junta de Andalucía y la llegada de José Rodríguez de la Borbolla, Presidente que tuvo a bien designarme Consejero de Cultura. Pero, en este contexto ¿cuál es el verdadero elemento de cambio? Me parece central un hecho que viene a marcar el fin de una etapa que podemos calificar de etapa épica de la Autonomía, la etapa de la gran movilización de los andaluces por conseguir el máximo nivel de autogobierno y que se había plasmado en una sucesión de victorias: se había ganado el 28 de febrero, se había ganado un Estatuto, se había constituido el primer Parlamento y el primer Gobierno Andaluz de la historia, se habían negociado los primeros decretos de transferencia... Los que venían detrás tendrían que buscar la legitimidad de la Autonomía no ya en ese poder carismático en el que se había basado el propio proceso de Rafael Escudero, ni en el discurso que permitió ganar el 28 de febrero o que permitió construir el discurso de la igualdad frente a otras Comunidades, sino que tenían que buscar la legitimidad de la Autonomía en hacer efectivo el autogobierno. La Autonomía sólo se legitimaría por el desarrollo eficiente y eficaz de las transferencias -que iban a empezar a llegar-al servicio de los ciudadanos.

El segundo elemento de importancia es la publicación en el Boletín Oficial del Estado, dos meses después de mi llegada a la Consejería de Cultura, del Decreto de transferencia de las competencias de cultura a la Comunidad Autónoma Andaluza, un Decreto negociado por el Consejero anterior, Rafael Román. Esta publicación tiene un enorme valor simbólico, un valor simbólico que siempre he relatado con una anécdota: se publica el Decreto de transferencia en mayo de 1984, y un día, en la Sede de la Consejería de Cultura, en la calle Castelar, en la que nos encontrábamos entonces, veo que hay un gran trasiego de cajas y de papeles en el patio de la Consejería -los que conocen el edificio saben que es un edificio histórico que tiene un gran patio central-, muchas cajas y un camión de mudanzas en la puerta de la Consejería del que un grupo de gente iba sacando cajas, y cajas, y cajas, muchas, de verdad. A mí me pareció impresionante. Y pregunté qué era aquello; Bartolomé Ruiz, en aquel momento Director General de Bienes Culturales, me contestó: "Consejero, es el Patrimonio Histórico". Efectivamente, en aquellas cajas nos llegaban los miles de expedientes que la Dirección General de Bellas Artes del Ministerio de Cultura tenía, unos resueltos, otros sin resolver, y decían: "son suyos, es su responsabilidad". Lo que transferían en aquellas cajas era la responsabilidad sobre el Patrimonio Histórico Andaluz.

Es una anécdota que hoy podemos recordar incluso con gracia, pero les puedo asegurar que a mí me abru- 
maba, porque inmediatamente después vi cuáles eran los efectivos de la Dirección General de Bienes Culturales: el Director General y cuatro colaboradores.

Con ello se inicia una etapa, de 1984 a 1986, que yo calificaría como la etapa del voluntarismo, en un sentido positivo, porque queríamos acometer más de lo que podíamos, de lo que teníamos conocimiento o estábamos en condiciones de hacer. Esta actitud permitió que fuésemos sentando las bases del proceso, si bien en aquel momento nadie tenía en la cabeza el Plan General de Bienes Culturales, y más bien todo se reducía a un trabajo pedestre tendente a dar respuesta cada día a problemas que nos aparecían cada día y que nos sorprendían cada día.

Carecíamos, también, de recursos económicos. El presupuesto de la Consejería de Cultura de aquel año era de algo más de 6.000 millones de pesetas, incluidos en esta cantidad los gastos de la Consejería, el capítulo I íntegro. Patrimonio no alcanzaba los 500 millones de pesetas.

Y carecíamos -fue éste uno de los primeros elementos que pudimos constatar- de personal especializado en el campo patrimonial. El método en el que se habían producido las transferencias había traído consigo unos Servicios Centrales de la Consejería sin historiadores, arqueólogos, arquitectos que hubieran orientando su trabajo hacia el terreno de los Bienes Culturales.

Como Administración nueva, emergente, que acababa de aparecer, carecíamos de autoridad, en el sentido de aceptación y respeto, carecíamos de autoridad y prestigio. Corría por aquella época una expresión muy común para calificar a los Delegados de la Junta de Andalucía; eran los chirrines, vocablo en origen definidor de los guardias forestales antiguos, pero ahora utilizado peyorativamente. Y esa Administración nueva tenía que hacer frente a Administraciones fuertes e históricamente consolidadas, como los Ayuntamientos y la Administración periférica del Estado, que venía de una larguísima tradición centralista que daba lugar a situaciones paradójicas, por ejemplo que el Director de una Institución cultural, de un Museo -lo digo con mucho respeto a todos ellos- podía ser en la vida local de una ciudad más importante que el propio responsable de la Administración Autonómica.

Con las transferencias tampoco nos llegó un diagnóstico de la situación, ni siquiera una advertencia del tipo "les puede ocurrir...", "les puede pasar...". Hoy, y esta fue nuestra experiencia, descubríamos por informes técnicos que el Museo de Bellas Artes de Sevilla se podía hundir. Mañana, que la sala principal de un Museo Arqueológico de Córdoba estaba en pésimas condiciones y que la cubierta, deteriorada, podía caerse en cualquier momento. El otro día, un periódico local muy interesado en la materia del Patrimonio nos revelaba en su primera página que el Palacio de Altamira ardía por la desidia de la Administración. Otro, nos podía sorprender con que un delegado había encontrado una caja de zapatos en la Alhambra con dos millones de pesetas sin ningún tipo de control o fiscali- zación previa, o que un Alcalde muy importante había decidido por su cuenta, sin encomendarse a ninguna de las medidas de tutela de la vida del Patrimonio, autorizar una urbanización en el entorno de la Alhambra. Otros alcaldes se proponían restaurar un edificio histórico catalogado, joya del barroco jerezano, en un proyecto sin ningún tipo de control administrativo, o hacer un aparcamiento subterráneo donde se encontraba uno de los yacimientos arqueológicos más importantes de la ciudad.

Y esto, les digo, nos sorprendía cada mañana, en una etapa de voluntarismo en la que no sólo queríamos resolver estos problemas que iban surgiendo, sino que en todos nosotros se iba creando una idea a la que urgente y necesariamente queríamos anticiparnos, una necesidad imperiosa de prever mínimamente lo que nos podía venir mañana, de tener preparada una respuesta.

Al respecto, y en mi opinión, el caso de la Alhambra -paradigmático en el modo de gestión de un conjunto monumental de esas características- tuvo una importancia decisiva en el modelo que implantamos después en otras Instituciones de la Comunidad. A muchos, encontrar un modelo de gestión -un buen modelo, creo modestamente- para la Alhambra nos llevó quebraderos de cabeza de carácter político y de todo tipo. La anécdota de la caja de zapatos que les contaba antes ocurre allá por 1985 y viene a evidenciar la quiebra y la falta de control de un modelo de gestión que confundía la gestión profesionalizada que debe de tener un monumento con la participación o las instituciones de participación de representación política o social que en un monumento como ese pueden concurrir. El Patronato era la gestión, y la gestión era el Patronato, pero como el Patronato eran personas de gran relevancia social no estaban en la gestión, y por lo tanto la gestión no estaba controlada por nadie, era un quehacer diario de quienes trabajaban allí, con su leal saber y entender. Les haré un reconocimiento. Yo nunca quise políticamente el conflicto de la Alhambra, me sobrevino y desbordó mis posibilidades; quise arreglar su gestión desde el principio, pero me encontraba con un grave problema: la Alhambra se había convertido en el símbolo de las transferencias de Patrimonio a la Comunidad. De hecho, el Decreto de transferencias no se cierra justamente porque no hay un acuerdo con la Alhambra, y precisamente éste fue el último hito de acuerdo que garantiza el paso de las transferencias de Cultura a la Comunidad. Ahora bien, ese acuerdo se hace, desde mi punto de vista y lo digo ahora aquí -se lo he comentado también en alguna ocasión a la Consejera- saltando la previsión constitucional. Yo intenté arreglar el acuerdo que aparecía en el Decreto de transferencias, pero no pude. La Alhambra tenía que ser transferida a la Comunidad Autónoma porque no había ninguna previsión estatutaria o constitucional que lo impidiera, y por lo tanto, como las competencias exclusivas en materia de Patrimonio Histórico eran de la Comunidad Autónoma, la Alhambra -su responsabilidad, claro, no su propiedad- tenía que ser transferida con las cajas, como todo lo demás. Pero había un modelo de gestión de Patronato estatal, presidido por unas personas nombradas por la Administración Central del Estado, de manera que 
tuvimos que hacer una transición para sustituir ese Patronato, y asumir la gestión de la Alhambra... aunque el Estado, en el Decreto de transferencia, se había reservado un Consejo, el Consejo de la Alhambra y Generalife, como órgano de tutela de la gestión de la Comunidad Autónoma. Se trataba de un elemento de control, en unos años (1984-1986) en que los grandes prebostes de la cultura española tenían escasa o nula confianza en la capacidad de las Comunidades Autónomas, creían que todo iba a ser un desastre, que íbamos a vender la Alhambra, o destrozarla.

Eso es lo que yo me encuentro cuando llego a la Consejería. El lío de la caja de zapatos, los dos millones, una Administración que no funciona... y alcanzo un acuerdo con el Ministro de entonces, Javier Solana, consistente en una postura intermedia, ante las alternativas de entender la creación del Consejo como una agresión a la Comunidad Autónoma (su creación nos hubiera llevado al Tribunal Constitucional) y su derogación total como una falacia del proceso de transferencia. Así que se optó por que la Junta de Andalucía regulara la gestión de la Alhambra y el Generalife mediante un modelo que separara claramente la gestión profesionalizada del monumento de la participación y la representación de las Instituciones y de las personalidades en la dirección del Patronato. Ese acuerdo se firmó, y también el Decreto que regula la Alhambra.

La búsqueda, pues, de una solución para la Alhambra nos marcó un criterio para la defensa de un modelo de gestión de los conjuntos monumentales, de los monumentos y otros centros y entidades que después aparecieron de la Comunidad. Y ese modelo se caracterizó por separar drásticamente la profesionalización de la gestión y la representación o dirección política, y por buscar instrumentos de autonomía, de gestión, para esos centros.

En la Alhambra este procedimiento fue sencillo porque era Organismo Autónomo, de modo que hoy se gestiona de una manera muy profesionalizada, con un Director al frente, nombrado por la Consejería de Cultura y del que depende una estructura fuerte de profesionales, en mi opinión bastante cualificada. Creamos asimismo un órgano de participación y de representación política en el que se encuentran la Consejería de Cultura, el Ayuntamiento de la ciudad, la Administración Central del Estado... e incluso pueden sumarse personalidades insignes. Pienso en definitiva, personalmente, que sigue siendo uno de los monumentos mejor gestionados en nuestro territorio.

Recuerdo aquel periodo, el de 1984 a 1986, los años del voluntarismo, como los años más duros, los más difíciles de toda mi etapa en la Consejería y probablemente en la política. El periodo 84-86 fue muy complicado, trabajábamos mucho, yo diría que más de doce horas diarias, y recogíamos poco. Esto en política es letal, en política hay que recoger, al menos lo suficiente para poder seguir avanzando. Pero en aquellos años los resultados de lo que hacíamos, su visualización, eran siempre en términos conflictivos o en términos de problemas. Conflictivos porque la Consejería se en- frentaba a Ayuntamientos como el de Granada, Córdoba, Jerez o Málaga -gobernados por Alcaldes de un enorme liderazgo social en sus ciudades- o problemáticos porque se nos caían edificios, se nos hundían artesonados, y se nos sublevaba el personal del entorno.

Pero de esa situación empezaron a surgir las ideas que permitieron después un periodo a mi parecer más fructífero. De esa situación fue apareciendo, junto a los problemas, una necesidad de abordar esos problemas, de enfrentarse a ellos, de no rehuir de ellos, y de elaborar una alternativa. Fuimos aprendiendo, por ejemplo, que era difícil acometer una tarea sobre el Patrimonio si no lo conocíamos, que la necesidad del diagnóstico pasaba a ser imperiosa, que había que conocer el estado del Patrimonio.

Así nos encontramos con un corolario, que en Andalucía la inmensa mayoría del Patrimonio cultural estaba en manos de la Iglesia católica. Yo aposté entonces por una opción, tan válida como otras, que consistía en establecer un acuerdo con la Iglesia -que resultó muy fructífero- con objeto de poder llegar a un conocimiento y diagnóstico de su Patrimonio. Por aquí veo a Reynaldo Fernández Manzano, que, como Director General de Instituciones del Patrimonio Histórico, fue un beneficiario indirecto de este acuerdo, porque le permitió adentrarse en el conocimiento del Patrimonio musical de la Iglesia, hasta ese momento cerrado a cal y canto. Fuimos llegando así a acuerdos en el terreno, nosotros invertíamos en el Patrimonio de la Iglesia, ellos garantizaban su uso público, o cedían a la Comunidad alguna parte.

Otra cuestión por abordar fue la dotación de personal especializado. En este sentido me ayudó bastante Bartolomé Ruiz, Director General de Bienes Culturales que, como arqueólogo, se aproximaba mejor que yo a las necesidades reales. No obstante, hay que recordar que nos encontrábamos con el grave problema de la escasa especialización del personal debido a una decisión tomada en la etapa inicial de la Autonomía y, desde mi punto de vista, errónea: como ningún funcionario especializado de los servicios centrales del Estado quiso irse a una Comunidad Autónoma se creó el incentivo de "la oferta pública de empleo", de modo que quien se pasase a la Comunidad mejoraba un grado, si era auxiliar pasaba a jefe de negociado, si era jefe de negociado pasaba a jefe de sección, si era de sección, de servicio... La necesidad de dotarnos de personal especializado fue apareciendo como una necesidad angustiosa, e intentamos resolverla de dos maneras: mediante la contratación externa y mediante un plan que inventamos entonces, el Plan Andalucía Joven ( $y$, dentro de él, el Plan de Bellas Artes) que permitió que muchísimos jóvenes andaluces se incorporaran a trabajar en estas materias. Inventamos, de alguna manera, las profesiones al hacerlas necesarias.

Se hacía también imperioso el incremento de los recursos económicos. Había poco dinero, necesitábamos más, y había que convencer al Gobierno. Pero todo Gobierno, y voy a hacer aquí una reflexión personal algo arriesgada a pesar de que veo por aquí a algún pe- 
riodista, todo Gobierno, en el mío también, aquel al que yo pertenecí, ha tenido la sensación de que el gasto cultural es como un gasto suntuario, que el gasto cultural es como el gasto que adorna el presupuesto, que nos permite, secundariamente, por ejemplo, un Museo en buenas condiciones. Yo empecé entonces una batalla, que después han continuado otros, y siguen ahí con ella, que venía a poner de manifiesto que esta concepción era un profundo error. Me habían impactado en mi periodo de Consejero de Cultura los viajes, bastantes, que hice a Italia, no sólo por su Patrimonio histórico envidiable sino porque habían aprendido a vivir de él, habían sabido sacarle rentabilidad. Destacaban, por ejemplo, ciudades que habían construido modelos de desarrollo económico basado, parcialmente, en un Patrimonio histórico bien conservado, bien divulgado y bien explotado en el sentido positivo de la palabra.

Poco a poco fuimos convenciendo de esta mentalidad y también fueron mejorando los resultados económicos, gracias en parte a una pequeña trampa que hacía yo por entonces: tuvimos unos inviernos muy lluviosos, el del 85, y el del 86, las cubiertas de nuestros monumentos se resentían muchísimo -y el mal de los monumentos empieza siempre en las cubiertas (eso lo aprendí de Román Fernández-Baca), de las cubiertas va bajando, va bajando, llega la humedad y ya no hay arreglo posible- así que empecé a utilizar una técnica, la declaración de emergencia. De este modo fuimos consiguiendo aumentar los recursos.

Tanta necesidad de respuestas nos abrumaba, a mí y al personal de la Consejería. No obstante, de este período 1984-1986 en el que buscábamos soluciones parciales a un problema, fuimos llegando a otro en el que maduró una idea de necesidad de previsión y que sirviese, me sirviese a mí para convencer al Gobierno de que tenía que apostar decisivamente por la Cultura.

Y esta etapa se cierra con un último acto de voluntarismo por mi parte, que es la inclusión en el programa electoral del Partido Socialista de 1986, como compromiso electoral, la elaboración de un Plan General de Bienes Culturales. Les confieso, eso sí, que cuando lo incluimos lo único que teníamos detrás de esa idea era la ambición de hacer un Plan que nos permitiese adelantarnos al tiempo, que nos permitiese fijar el diagnóstico de la situación, saber hacia dónde queríamos llegar y poner los medios para lograrlo. Pero no teníamos ni mucho menos en ese momento la idea de lo que luego resultó ser.

Las elecciones del 86 confirman la mayoría del Partido Socialista y del presidente Borbolla, que a su vez me confirma como Consejero de Cultura, y afronto una etapa, una segunda etapa, desde mi punto de vista no más creativa que la anterior, pero sí quizás con mejores -o más visibles- efectos.

Partimos de una indefinición inicial, no sabíamos qué hacer, les vuelvo a repetir, con el Plan. Tuve conversaciones con el Director General de Bienes Culturales de entonces, Bartolomé Ruiz, para decidir si hacíamos un Plan que sólo abarcase la conservación y la restauración (era el tema que más nos preocupaba, especialmente en lo referido a la situación de los inmuebles) o que se acercara a la distribución del Patrimonio, que era también tremenda, con una situación jurídica muy compleja y que aún hoy, lamentablemente, no se ha resuelto. Y digo lamentablemente pensando en cartas de aquella época dirigidas por mi parte tanto a Javier Solana como a Jorge Semprún diciéndoles que era un despropósito el modelo de gestión transferida a la Comunidad de las Instituciones del Patrimonio con titularidad estatal, porque impedía abordar los problemas de fondo.

Definitivamente, creo que hay un hecho que podemos reconocer como desencadenante último del Plan. Se trata del hundimiento de la Biblioteca Capitular y Colombina en 1987, que viene a suponer el punto final de esos derrumbes de que hablaba antes. Se nos caía la Catedral de Sevilla, e inevitablemente esto nos llevaba a tener que dar una respuesta política inmediata y cercana. Recuerdo vagamente una reunión del Consejo de Dirección, una entrevista con el Director General... el Plan se hacía ya inaplazable y tenía que estar listo en el menor tiempo posible. Recurrimos a la contratación externa, y en este punto quiero mencionar a una persona que ya no está entre nosotros, pero a la que yo le debo mucho, una persona que fue un buen consejero personal, un buen amigo, y ayudó mucho en temas trascendentales del Patrimonio de nuestra Comunidad; me estoy refiriendo a Fernando Villanueva, a quien encargué la hilvanación del Plan General de Bienes Culturales y el comienzo de su andadura. Adoptamos una decisión, que el Plan tenía que ser un elemento estratégico que abarcara la totalidad de la política de Bienes Culturales desde la investigación, la conservación, la difusión, las instrucciones culturales y la administración del Patrimonio, porque teníamos la obsesión de incentivar la Administración de Cultura. No estaba en ese momento inicial el Instituto Andaluz del Patrimonio Histórico, y les voy a explicar por qué.

El modelo que nosotros veníamos discutiendo, el debate que teníamos sobre la manera de la intervención, de la investigación, de la restauración de los Bienes Culturales partía de los elementos centrales, de los propios museos. En nuestro primer modelo queríamos que los museos se dotaran, en función de su importancia, de laboratorios de análisis y de personal especializado para que fuesen contribuyendo a la restauración, a la investigación, porque habíamos aprendido en este proceso de ir interviniendo cada vez más y destinando más recursos económicos al Patrimonio que el Patrimonio era el pasado, pero que si no nos apuntábamos a los nuevos hallazgos, a las nuevas tecnologías, a las nuevas técnicas que se estaban desarrollando, nos íbamos a quedar atrás. En este sentido, nuestro primer modelo hacía de los museos la cabecera de esta cuestión.

Pero el hundimiento de la Colombina también nos ayudó a despejar esta incertidumbre. El modelo de los museos cabecera para la investigación y la difusión culminaba con el Instituto Español del Patrimonio Históri$\mathrm{co}$, creado en el Ministerio de Cultura, y, a nuestro juicio, último recurso para aquellas intervenciones que 
superaran el ámbito de actuación propia. Tuve varias reuniones en relación con algunos incunables de la Colombina, y les puedo decir que la experiencia fue un desastre. Mi recuerdo personal de aquella experiencia es que no se obtenía respuesta, no existía capacidad técnica en aquel momento (no conozco la situación actual) y había una cierta mirada distante hacia estos chicos que vienen de la Autonomía. Bueno, visitamos entonces dos modelos, el italiano (no olvidaré nunca la imagen del patio con el Marco Aurelio en proceso de restauración) y otro bien diferente, el de Bruselas, con una capacidad tecnológica importante. Decidimos, a la vuelta de estas experiencias, negativa en el caso español, positiva en los casos italiano y belga, la inclusión del Instituto Andaluz del Patrimonio Histórico como una institución cúspide en el marco del Plan General de Bienes Culturales encargada de nuestra I+D, que se dedicara a la investigación, a la difusión, a la investigación aplicada a la restauración y a la recuperación de piezas notables de nuestro Patrimonio que requiriesen un tratamiento específico.

Con el Instituto experimentamos un modelo que se le había ocurrido, como modelo administrativo, al Director General de Deporte. Sé que esto le suscita mucha risa a Román Fernández-Baca, pero, aunque yo quería reproducir el modelo de la Alhambra en las Instituciones del Patrimonio de Andalucía, era imposible la creación de un organismo autónomo, puesto que la Consejería de Economía y Hacienda en aquellos 1988-1989 era muy reacia a la creación de estas entidades, consideradas como una fuga de los controles de la Administración. Así que, como decía, alguien en la Dirección General de Deportes, por otras necesidades, que eran muchas, creó una entidad que se llamaba Unisport, basándose en una vieja ley del 58, la Ley de Entidades Administrativas Autónomas, que contemplaba una figura administrativa que se ha conocido después como el cero cuatro (los 0.4. son servicios sin personalidad jurídica pero con funcionamiento autónomo) que garantizaba la creación de un Instituto con cierta autonomía de gestión, lo que nos posibilitaba el objetivo de una profesionalización a ultranza de la institución, que dependía de la centralización y jerarquía de la Consejería de Cultura a través de la Dirección General de Bienes Culturales. Hay que reconocer, pues, la ocurrencia de este modelo al personal de Deportes, un modelo que se fue imponiendo y aplicando en Cultura, y que encontró su plena potencialidad en las Instituciones del Patrimonio, no sólo en el Instituto sino también en los otros Centros de la Comunidad.

Decidida por tanto la creación del IAPH, elegido el modelo administrativo, el tercer elemento era la sede. Y como casi todo de aquella etapa, el resultado fue el resultado de un conflicto.

En esta ocasión, la disputa se establece en el año 86-87 entre la Consejería de Obras Públicas de la Junta de Andalucía -a la que el Decreto de transferencias había atribuido el edificio que es sede actual del Instituto Andaluz del Patrimonio Histórico, porque pertenecía a la Dirección General de Arquitectura del Ministerio- y la Consejería de Cultura -que reclamaba este edificio porque era un Bien de Interés Cultural y por lo tanto tenía que hacerse responsable de su restauración. En el fondo de la batalla, como en otras muchas, se libraba una cuestión económica; si recibíamos el edificio nos vendría el dinero para su rehabilitación, y si nos venía el dinero para su rehabilitación nos llegaría también la capacidad de influir en el tema principal de las tertulias sevillanas del momento: la Exposición Universal, en cuyo corazón se hallaba el edificio. Lograr el edificio significaba que la Administración de Cultura iba a poder decir también algo en el diseño de la Exposición.

Conseguido finalmente el edificio, surge la necesidad de llenar de contenido el espacio, de hacer que el debate que se había suscitado no acabara siendo meramente retórico. Si habíamos luchado tanto por dirigir la rehabilitación de un edificio, si habíamos peleado por decir que la orientación de esa rehabilitación debía ser básicamente cultural, teníamos el deber de hacer una propuesta de contenido, en la que aparece, en primer lugar, la ubicación del Instituto Andaluz del Patrimonio Histórico en la zona fabril que hoy ocupa, afortunadamente.

Resuelta la necesidad, el modelo y la sede, se creó el Instituto Andaluz del Patrimonio Histórico, que hoy celebra diez años.

Yo, por estos diez años, y para terminar ya, les quería decir en primer lugar felicidades. Creo que tras este periodo el Instituto Andaluz del Patrimonio Histórico es una institución de prestigio, que con su prestigio ha prestigiado a la Comunidad Autónoma que lo ideó. Es el resultado de una ambición, de una ambición política, y, si lo desean, de la ambición de una generación que hizo de su ambición política el hacer útil, de una generación a la que yo, finalmente, tengo que estar agradecido. Una generación de políticos - desde el Presidente que me nombró hasta otros muchos que espero se sientan representados aunque no los pueda citar a todos: Luis García Garrido, aquel Viceconsejero que nos ayudó a resolver y a quitar papeles, materia en la que no éramos muy expertos; Bartolomé Ruiz, el primer Director General de Bienes Culturales; Pepe Guirao, que culmina la definición de la gestión del Instituto; Mateo Revilla, Pedro Navarro, y tantos otros- $y$ de profesionales y funcionarios, entre los que destaco a dos que simbolizan a todos: Fernando Villanueva, que ya he nombrado, y Pedro Salmerón, quien llevó adelante el final de la tarea.

También, y sobre todo, a Román Fernández-Baca, por hacer el Instituto en el que él creía. Yo pienso que ha acertado, pues, como ha escrito Angelo Bianco, estudioso italiano de la vida de las organizaciones y de los partidos políticos, la clave para comprender el desarrollo futuro de una organización está en su momento fundacional, puesto que este momento marca tal impronta que transciende mucho más allá en el tiempo.

Por supuesto, termino ya, con el agradecimiento a los que me siguieron, sin ellos el Instituto no cumpliría diez años: a Juan Manuel Suárez Japón, a José María Martín Delgado, y a Carmen Calvo, que hoy conmemora el cumpleaños. A todos ustedes muchas gracias. 